\title{
A phase I Study with oral SU5416 in patients with advanced solid tumors: A drug inducing its clearance
}

\author{
Marc Salzberg ${ }^{1}$, Miklos Pless ${ }^{1}$, Christoph Rochlitz ${ }^{1}$, Klara Ambrus ${ }^{1}$, Paul Scigalla ${ }^{2}$ and \\ Richard Herrmann ${ }^{1}$ \\ ${ }^{1}$ University Hospital Basel, Switzerland; ${ }^{2}$ Pharmacia Inc., Peapack, USA
}

Published online: 5 October 2005

Key words: SU5416, anti-angiogenesis, inhibition neovascularization, VEGF, tyrosine kinase, Nanocrystal Colloidal Dispersion.

\begin{abstract}
Summary
Vascular endothelial growth factor (VEGF) is a potent stimulant of angiogenesis. SU5416, is a small molecule tyrosine kinase inhibitor, and a potent inhibitor of VEGF- mediated Flk-1 receptor signaling. Intravenous agent SU5416 has shown evidence of biological activity against a variety of tumor types. The current intravenous dosing regimen is not optimal for long-term administration, which is needed for optimal efficacy. The aim of this study was to evaluate the safety profile and pharmacokinetics of a Nanocrystal Colloidal Dispersion ${ }^{\mathrm{TM}}$ (NCD) SU5416 formulation in humans. Patients with advanced and/or metastatic solid organ tumors were included in the trial; various SU5416 regimens were tested for tolerability, safety and were evaluated concerning pharmacokinetics. The results of this study indicate that induction of clearance after oral dosing of NCD SU5416 in humans occurs and is greater than following IV administration. It has been confirmed that SU5416 is a high clearance compound, also as an oral NCD formulation. The NCD formulation was well tolerated, but no effective drug serum levels could be achieved. These data help to understand the ADME (Absorption, Distribution, Metabolism, Excretion) properties of indoline chemical class compounds. The lessons learned should be applied in the development of next generation indoline anti-angiogenic and anti-tumor compounds.
\end{abstract}

\section{Introduction}

New blood vessels are required for the growth of solid tumors beyond a volume of $1-2 \mathrm{~mm}^{3}$. Tumors that lack adequate vasculature undergo hypoxia, and become consequently necrotic or apoptotic [4]. Tumors that undergo neo-vascularization not only enter a phase of rapid growth but also demonstrate increased metastatic potential. Immunohistochemical studies on tumor sections from margins of growing tumors show a preponderance of blood vessels, irrespective of tumor type. This preponderance of blood vessels is a result of angiogenesis triggered by factors released from hypoxic tumor cells that migrate to nearby blood vessels and stimulate growth of endothelial cells. One of the most potent stimulants of angiogenesis is vascular endothelial growth factor (VEGF) [2]. Data concerning the expression of VEGF in colorectal cancer demonstrated a correlation of progression of disease with VEGF expression and it also showed to be an inde- pendent prognostic factor in colorectal cancer and other malignancies [3, 4].

Inhibition of neo-vascularization driven by VEGF appears to be a promising approach to reduce or prevent tumor re-growth or proliferation of metastases in advanced solid tumors $[5,6]$. The search for the receptor whose selective inhibition will prevent new blood vessel growth has been the focus of basic research for the last ten years. Although there are multiple receptors whose expression is restricted to endothelial cells, it is believed that the Flk-1 receptor plays a role in angiogenesis and thus, its inhibition may have therapeutic benefit for cancer patients.

SU5416 (Z-3[(2,4-dimethylpyrrol-5-yl)methyldenyl]2-indolinone; generic name: semaxanib), is a small molecule tyrosine kinase inhibitor. In vitro, SU5416 inhibits Flk-1 receptor phosphorylation with an $\mathrm{IC}_{50}$ of approximately $1 \mathrm{mM}$. It inhibits VEGF-stimulated proliferation of endothelial cells with $\mathrm{IC}_{50}$ values of approximately $0.04 \mathrm{mM}$. The inhibitory effects of SU5416 
are time dependent. This molecule has been primarily studied as an intravenous agent in patients with a variety of advanced malignancies and has shown preliminary evidence of biological activity against a variety of tumor types as an intravenous agent [7]. However, it seems that angiogenesis inhibitors require long-term administration for optimal efficacy. The current intravenous dosing regimen, twice-weekly infusions, is rather cumbersome and probably not optimal for long-term administration. In addition, SU5416 is classified as a high-clearance compound following IV administration [8].

The aim of this study was to evaluate the safety, toxicity, and pharmacokinetics of a Nanocrystal Colloidal Dispersion (NCD) SU5416 formulation in humans. If the safety and pharmacokinetic (PK) profile of the single dose proves to be acceptable, a variety of dosing regimens were to be explored to determine the impact of repeat dosing on the pharmacokinetics of oral SU5416.

\section{Patients and methods}

\section{Patient selection}

Patients were eligible if they were at least 18 years of age with histologically proven advanced solid tumors. They could have received one or more prior systemic chemotherapy regimen, provided that the treatmentfree interval was $\geq 4$ weeks ( $\geq 6$ weeks in case of mitomycin-C and nitrosourea) and that it did not contain high-dose chemotherapy. Patients agreed to use effective contraception, if applicable. All at-risk female patients were required to have a negative serum pregnancy test within 7 days prior to study drug administration. Patients were required to have a life expectancy of at least 12 weeks, an Eastern Cooperative Oncology Group (ECOG) performance status of $\leq 2$ and adequate hematological reserve (absolute neutrophil count (ANC) $>1,500 / \mathrm{mm}^{3}$; hemoglobin $>10 \mathrm{~g} / \mathrm{ml}$; platelet count $>100,000 / \mathrm{mm}^{3}$ ), no significant liver function abnormality (bilirubin $\leq 35 \mu \mathrm{mol} / \mathrm{L}$, and/or serum transaminases, alanine aminotransferase (ALT) and aspartate aminotransferase (AST) $<3.0$ upper limit of normal) and no significant renal function abnormality (serum creatinine $<160 \mu \mathrm{mol} / \mathrm{L}$ or creatinine clearance $<50 \mathrm{~mL} / \mathrm{min}$ ). Patients with a history of myocardial infarction in the past 12 months, severe or unstable angina pectoris, and patients, who underwent coronary or peripheral artery bypass surgery within the last two years were excluded. The latter could be enrolled after consultation with a cardiologist confirming that the disease was stable.

Written informed consent was obtained from each patient. The protocol, patient information and informed consent form were approved by the local ethics committee and notified by the national regulatory authorities.

\section{Treatment and $P K$-sampling plan}

In the first stage of testing, 5 patients received a single intravenous dose of SU5416 on day 1. On day 8, each patient received a single dose of oral NCD SU5416. The $\mathrm{IV}$ and the oral doses were $75 \mathrm{mg}$ per administration. The half-life for the IV-formulation is approximately 55 minutes [9]. It was estimated that 3-6 patients were sufficient per dose-level to assess the bioavailability of the oral formulation. Plasma samples were collected on day 1 and on day 8 for PK analysis at the following timepoints:

- IV: Before infusion, immediately prior to EOI, 5, 10, 20, 30, 45, 60, 120, 180, 240 following infusion.

- PO: Before ingestion, 30, 45, 60, 90, 120, 180, 240 following ingestion.

The same timepoints were applicable for the second stage as well.

In the second stage of testing, patients $(n=3$ per group; if greater than $30 \%$ variation in bioavailability between patients was observed, the group size was to be increased to 6) were to receive an initial intravenous dose of SU5416 on day 1 as a control and oral doses of NCD SU5416 starting on day 8. Based on safety and PK data observed in the first stage of testing, different repeat-dose regimens were to be tested, including weekly dosing (Days 8, 15, and 22 ), twice weekly dosing (Days 8, 11, 15, 18, and 22), and daily dosing (Days 8 through 21). Dose escalation (from 75 to 150 to $300 \mathrm{mg}$ daily dose) was to occur for 1 of these regimens based on the most frequent administration consistent with acceptable safety and PK parameters. Dose escalation was to discontinue if there were 2 or more patients in a dose group with unacceptable toxicites being defined as:

- toxicity grade change in ALT, AST, alkaline phosphatase or bilirubin lasting $>7$ days (possibly or probably related to drug)

- toxicity grade change in diarrhea (>Grade 1) lasting more than 7 days with supportive care

- new onset change in parameters of thrombosis (PT or APTT)

- Serious Adverse Events or Grade 3,4 or fatal Adverse Event (possibly or probably related to drug).

Patients were to be screened within 14 days prior to study drug administration and drug levels to be evaluated for toxicity from the first day to the last day of dosing plus at a follow-up visit 7 days following their last dose of drug. Patients with no signs of progressive disease were given the option, at the discretion of the treating physician, to receive IV SU5416 as compassionate use treatment. 
The protocol called for the use of dexamethasone and H1- and H2-blockers as pre-medications for the SU5416 infusion. All 14 patients (100\%) used dexamethasone, $\mathrm{H} 1$-antagonist (Clemastine) and H2-antagonist (Ranitidine) as premedications. SU5416 was administered via a venous access device.

For PK the following procedure was applied:

1. $4 \mathrm{ml}$ blood was collected in a vacuum tube containing heparin; tube was gently inverted 15 times in order to completely mix blood and heparin; specimen was placed on ice; tubes had to be kept at $2-8^{\circ} \mathrm{C}$ at all times.

2. Samples was centrifuged: $3500 \mathrm{rpm}$ at $4^{\circ} \mathrm{C}$ for 10 minutes

3. Upper layer was transferred with a glass pipette to a Nalgene cryovial $(1.5 \mathrm{ml})$

4. Tubes were stored at $-70^{\circ} \mathrm{C}$ and sent packed in dry ice to Specialty Laboratory in Santa Monica, California for analysis.

\section{Bioanalytical methods}

SU5416 plasma concentrations were determined using a validated HPLC assay with UV detection by Specialty Laboratories. Two oxidative metabolites, an alcohol designated SU9838 and a carboxylic acid designated SU6595 were determined. Aliquots of plasma samples $(200 * \mathrm{~L})$ were acidified by the addition of $1 \mathrm{M}$ hydrochloric acid $(10 * \mathrm{~L})$. The internal standard SU5614 $(5.0 \mathrm{mg} / \mathrm{mL}$, $50 * \mathrm{~L}$ ) was added and compounds of interest extracted by the addition of acetonitrile $(1.5 \mathrm{~mL})$. The mixture was centrifuged, the organic layer decanted from the precipitated proteins and evaporated to dryness under dry nitrogen. The residues were reconstituted with DMSO: $\mathrm{CH}_{3} \mathrm{CN}$ :Mobile Phase, 2:4:3 vol:vol and injected onto a reverse-phase column. The mobile phase gradient consisted of $10 \mathrm{mM}$ ammonium formate $\mathrm{pH}=2.6$ and acetonitrile over 30 minutes at a flow rate of $1.2 \mathrm{~mL} /$ minute. The column eluant was monitored with a UV/visible detector set at $440 \mathrm{~nm}$.

The assay is linear from 10 to $2000 \mathrm{ng} / \mathrm{mL}$. Intraassay coefficients of variation fall between 2 and $10 \%$ tested at low $(100 \mathrm{ng} / \mathrm{mL})$ medium $(500 \mathrm{ng} / \mathrm{mL})$ and high $(2000 \mathrm{ng} / \mathrm{mL})$ plasma concentrations. Inter-assay coefficient of variation is between 3 and $6 \%$ over the same range of concentrations; accuracy is 94 to $99 \%$. Quality control samples at 3 different concentrations were developed to be included with each analytical run and monitored using Westgard rules in compliance with Good Laboratory Practice. The lower limit of quantitation for SU5416, SU6595 and SU9838 were 10, 8 and $18 \mathrm{ng} / \mathrm{mL}$, respectively.

\section{Pharmacokinetic analysis}

SU5416 PK parameters were calculated using noncompartmental methods using Kinetica (Version 3.1, InnaPhase, Corp). The primary PK parameters evaluated for SU5416 were $C_{\max }$ and AUC. In addition, the secondary PK parameters were also evaluated: $T_{\max }, \mathrm{CL}, \mathrm{Vz}$, $\mathrm{t} 1 / 2$, and oral bioavailability. The following pharmacokinetic parameters were determined by noncompartmental methods:

$C_{\max }$ : Peak plasma concentration was identified by inspection of individual subject concentration-time curves.

$T_{\max }$ Time of observed peak plasma concentration was identified by inspection of individual subject concentration-time curves.

AUC0-tlast: Area under the plasma concentration-time curve from time zero to the time (T) of the last detectable concentration. AUC0-t(last) was calculated using the trapezoidal rule.

$\mathrm{V}_{\mathrm{z}}$ : Volume of distribution based on the terminal phase was calculated as $\mathrm{V}_{\mathrm{z}}=$ Dose/AUC $* \mathrm{l}_{\mathrm{z}}$; where $\mathrm{l}_{\mathrm{z}}$ represents the terminal slope

CL: Total body clearance was calculated as $\mathrm{CL}=$ Dose/AUC

t1/2,z: Terminal elimination half-life was calculated as $\mathrm{t} 1 / 2, \mathrm{z}=0.693 / \mathrm{lz}$, where the apparent elimination rate constant, $\mathrm{lz}$, was estimated by least-squares regression of values in the terminal log-linear region of the plasma concentration-time curves.

F(oral): Oral Bioavailability;

$$
\begin{aligned}
\mathrm{F}(\text { oral })= & \text { AUC0-tlast }(\text { oral }) \\
& * \text { Dose }(\mathrm{IV}) / \mathrm{AUC} 0 \text {-tlast }(\mathrm{IV}) * \text { Dose }(\text { oral })
\end{aligned}
$$

PK-parameters at the different timepoints were compared by calculating\%-age of deviation of the values. Analyses are descriptive in nature.

\section{Results}

The 14 patients who received SU5416 treatment comprised the intent-to-treat (ITT) population. Of these, five patients $(36 \%)$ were treated under Stage 1 (single dose of $75 \mathrm{mg}$ of oral SU5416). Nine patients (64\%) were treated under Stage 2. Of these, three patients $(21 \%)$ received weekly dosing of study drug (75 mg of oral SU5416 on days 8,15 and 22$)$; three patients $(21 \%)$ received twice weekly dosing of study drug (75 mg of oral SU5416 on days $8,11,15,18,22)$; and another three patients $(21 \%)$ received daily dosing of study drug (75 mg of SU5416 on days 8 through 21 ). One patient (7\%) discontinued the 
Table 1. Patient baseline and disease characteristics

\begin{tabular}{ll}
\hline Male/Female & $n=8 / n=6$ \\
Median age in years (range) & $51(25-69)$ \\
ECOG score & 13 pts. With ECOG 1 at baseline \\
Disease characteristics & Solid organ tumors. \\
& Non-small cell lung cancer $(n=4)$ \\
& Colorectal cancer $(n=2)$ \\
& Others $(n=8)$ \\
Previous therapies & Surgery: all patients, $n=14$ \\
& Radiotherapy: $n=11(79 \%)$ \\
& Systemic therapy: $n=13(93 \%)$ \\
\hline
\end{tabular}

study due to death. Thirteen patients (93\%) completed the study.

One patient $(7 \%)$ was enrolled in the study with violations of inclusion/exclusion criteria. A 69-year-old white male with a history of metastatic colon carcinoma had received surgery, radiotherapy, and systemic therapy. This patient had hemoglobin value of $<10 \mathrm{mg} / \mathrm{mL}$ at screening.

All analyses were performed using the data set that contained all data for all patients who received at least 1 dose of oral SU5416, the ITT population.

It became apparent that no effective drug serum levels could be achieved with the NCD-formulation, therefore the number of patients were not increased as originally foreseen in the study protocol. For the same reason, no dose escalation was performed.

\section{Demographic and other baseline characteristics}

Baseline characteristics of the 14 recruited patients are summarised in Table 1.

Patients were polymorbid and most of them had signs and symptoms caused by the advanced stage of their tumor. Consequently, all patients used concomitant medication.

\section{Dosage information}

All patients received $75 \mathrm{mg}$ IV SU5416 except 1 patient in the Stage 2, twice-weekly group who received $50 \mathrm{mg}$ inadevertently. All infusions were to be administered at a fixed infusion rate of $200 \mathrm{ml} /$ hour, which resulted in infusion times of 30-60 minutes. According to the infusion records, 5 patients had IV SU5416 infusions interrupted. In all these patients, infusions were interrupted due to AEs such as mild or moderate pain at the injection site.

All patients received oral SU5416 and all completed the schedules. All oral doses were $75 \mathrm{mg}$.
Table 2. SU5416 Mean ( \pm SD) Pharmacokinetic Parameters following IV Infusion of SU5416 (75 mg) and Single Oral Dose of SU5416 (75 mg)

\begin{tabular}{lcc}
\hline & & $\begin{array}{c}\text { Single Oral Dose } \\
\text { SU5416 (75 mg) } \\
\text { Parameters* }\end{array}$ \\
\hline$C_{\max }(\mathrm{ng} / \mathrm{mL})$ & $2810 \pm 1110$ & $233 \pm 543$ \\
$T_{\max }(\mathrm{minutes})$ & $\mathrm{NC}^{\dagger}$ & $67 \pm 26$ \\
$\mathrm{AUC}_{0-\text { tlast }}(\mathrm{ng} / \mathrm{mL} *$ & $1210 \pm 383$ & $220 \pm 340$ \\
$\quad$ hour) & & \\
Clearance (mL/min) & $47.5 \pm 23.2$ & $9.23 \pm 7.51$ \\
$V_{\mathrm{z}}$ (mL) & $2900 \pm 1430$ & $\mathrm{NC}^{\dagger}$ \\
Half-life (minutes) & $43.5 \pm 12.4$ & $\mathrm{NC}^{\dagger}$ \\
Bioavailability & $\mathrm{NC}^{\dagger}$ & $19 \pm 27.4$ \\
\hline
\end{tabular}

${ }^{*} C_{\max }=$ peak plasma concentration, $\mathrm{AUC}_{0-t \text { (last) }}=$ area under plasma concentration- time curve, $\mathrm{Vz}=$ volume distribution base on the terminal phase.

${ }^{\dagger} \mathrm{NC}=$ Not calculated.

\section{Pharmacokinetics}

The peak concentration and the area under the curve of the oral SU5416 equaled only approximately $10 \%$ of the values achieved following the IV administration of SU5416 (Table 2). The IV pharmacokinetics were very similar to those observed in previous studies.

Plasma concentrations were generally lower during Days 15 and 21/22 as compared to, Day 8. By Day 15, a number of patients' SU5416 concentrations were below the level of detection. Mean concentrations (Cmax) of oral SU5416 were approximately 12 -fold lower than those observed following IV infusions. The mean peak concentrations following a single oral dose of NCD SU5416 were observed at 67 minutes. The mean bioavailability for a single dose and weekly, twice weekly, and daily dosing of NCD SU5416 on day 8 were 18.9, 20.6, 20.7 and $36.8 \%$ respectively. However, there was a large interpatient variability. One patient had an oral bioavailability of $1.3 \%$ (Table 3 ).

The pharmacokinetics of NCD SU5416 following oral administration exhibited large inter- and intra-patient variability. With the oral formulation used in this study only $10-15 \%$ of plasma concentrations of SU5416 could be achieved following a single administration compared with the IV SU5416. In addition, the oral absorption of NCD SU5416 was highly variable ranging between 1.3 to $68 \%$. SU5416 concentrations were lower during Days 15 and 21/22 as compared to day 8 suggesting induction of clearance.

\section{Safety}

The oral SU5416 was generally well tolerated, and there were no apparent differences between groups in likelhood 
Table 3. Mean SU5416 ( \pm SD) pharmacokinetic parameters following three different oral dosing schedules

\begin{tabular}{lllll}
\hline Treatment & AUC Day 8 & AUC Day 15 & AUC Day 21/22 & Induction of clearance \\
\hline Oral & & & & \\
$\quad$ Once weekly $(n=3)$ & $156 \pm 117$ & $131 \pm 140$ & $141 \pm 90$ & $10 \%$ \\
$\begin{array}{l}\text { Twice weekly } \\
(n=3)\end{array}$ & $329 \pm 187$ & $117 \pm 92$ & $198 \pm 321$ & $40 \%$ \\
Daily dosing $(n=3)$ & $412 \pm 111$ & $21 \pm 36$ & $9 \pm 16$ & $98 \%$ \\
\hline
\end{tabular}

The calculation was made by comparison of the AUC measured on Day 8 with those on day 21/22.

of experiencing SAEs or treatment-related adverse events (see Table 4).

\section{Discussion}

A large inter- and intrapatient PK-variability has been observed. During metabolization SU5416 is converted to a number of metabolites, the pattern and levels of which vary markedly. Hepatic microsomes from humans form six metabolites. The predominate metabolite was identified as the primary alcohol SU9838, resulting from oxidation of 5-methyl group on the pyrrol ring SU5416. SU9838 accounts for approximately $41 \%$ of the total metabolites formed by human microsomes after a 15 minute incubation. Additional SU5416 microsomal metabolites were identified as further oxidative products of SU9838, e.g. the carboxylic metabolite SU6595, or products remaining from ring hydroxylation [10]. The conversion of SU5416 to SU9838 is primarily catalyzed by CYP1A2 with minor contributions from other enzymes. The formation of SU6595, appears to be entirely catalyzed by CYP1A2. A correlation analysis be- tween the CYP1A2 activity and the formation of SU9565, SU9838, or the sum of the two metabolites showed that SU9838 behaved as primary metabolite, in that its formation strongly correlated with CYP1A2 activity. These results suggested that CYP1A2 plays a major role in converting SU5416 to the carboxylic acid without release of intermediate alcohol metabolite. Thus, the metabolism of SU5416 by CYP1A2 undergoes "concerted" oxidation to the alcohol SU9838 and then to the carboxylic acid SU6595 [11].

In vitro liver microsomal analysis showed that metabolism of SU5416 increased by a factor 2-3 in animals receiving multiple IV doses of the drug. The activity of CYP1A2 increased two-folds in the treated group compared to the vehicle control group. Another study in dogs treated with SU5416 at $20 \mathrm{mg} / \mathrm{kg} /$ day orally for 14 days detectable plasma levels of SU5416 were seen on Day 1 of dosing, but on Day 13 of repeated dosing, no SU5416 was detectable in plasma. Liver tissue analysis showed that repeated SU5416 dosing produced an 17-fold increase in the activities CYP1A2, hepatic SU5416 metabolism was increased by at least 8 -fold [12]. A further in vitro study

Table 4. Summary of most frequent and most severe treatment-emergent adverse events and hematological toxicites with $\operatorname{SU} 5416(n=14)$

\begin{tabular}{llllll}
\hline Adverse event & Mild & Moderate & Severe & Life-threatening & Fatal \\
\hline Any ae & 5 & 5 & 2 & 1 & 1 \\
Vomiting & 6 & 0 & 0 & 0 & 0 \\
Nausea & 3 & 1 & 0 & 0 & 0 \\
Dyspepsia & 0 & 0 & 1 & 0 & 0 \\
Asphyxia & 0 & 0 & 0 & 0 & 1 \\
Hematoma & 0 & 0 & 1 & 0 & 0 \\
Back Pain or exacerbated pain & 0 & 1 & 2 & 0 & 0 \\
Injection site pain or burning & 4 & 3 & 0 & 0 & 0 \\
Weakness & 1 & 0 & 1 & 0 & 0 \\
Narcolepsy & 0 & 0 & 1 & 0 & 0 \\
Urinary Incontinence aggravated & 0 & 0 & 0 & 1 & 0 \\
\hline
\end{tabular}

Values reflect number of patients. 
confirmed that SU5416 produced a time and concentration dependent induction in the CYP1A2 promotor driven induction of luciferase activity in TV101 cells [13, 14].

Our study showed that induction of clearance after oral dosing of NCD SU5416 in humans occurs substantially and is even greater than following IV administration. However, a phase I study with once-weekly dosing of SU5416 maintains a comparatively higher systemic exposure for a given dose of that drug, as it prevents the induction in clearance seen with twice-weekly infusions and SU5416 [15]. A typical long-lasting inhibitory activity in vitro and in vivo was suggested for SU5416. It is preferentially concentrated in cells, and the cells maintain an inhibitory concentration of SU5416 for a prolonged period even when the compound is no longer present in the medium [16]. Less hydrophobic compounds such as SU6668 do not demonstrate long-lasting activity or concentration in similar studies [16].

It has been confirmed in this clinical trial that SU5416 is a high clearance compound, also as an oral NCD formulation. The development of that compound was not pursued, because it was unlikely that effective plasma concentrations could be achieved with this formulation, with SU5416 as such, and the data discussed in this paper help to understand the pharmacokinetic properties of indoline chemical class compounds. The lessons learned should be applied in the development of next generation indoline anit-angiogenic and antitumor compounds.

Repeated dosing of SU5416 markedly induced hepatic CYP1A activity in vivo, and this induction is most likely responsible for the attenuation of exposure observed in various species including humans upon repeated dosing.

There is an increase of certain AEs with SU5416 including vomiting, nausea, and injection site pain and burning. These are known and expected risks in relation to the administration of SU5416. The results of hematology, serum chemistry, urinalysis, vital sign measures, ECGs, physical examinations and toxicity evaluations did not indicate any unexpected risks for SU5416 administration. The oral NCD formulation of SU5416 was well tolerated and safe. It has to be taken in consideration, though, that only $10-15 \%$ of plasma concentrations could be achieved compared with the IV formulation. These levels are not likely to be therapeutic.

\section{References}

1. Folkman J: Antiangiogenic therapy. In: de Vita VT Jr, Hellman S, Rosenberg SA (eds.): Cancer: Principles and practice of oncology, 5th edn. Philadelphia, PA: Lippincott-Raven, 3075-3085, 1997
2. Berlin JD: Targeting vascular endothelial growth factor in colorectal cancer. Oncol Suppl 16(8): 13-15, 2002

3. Lee JC, Chow NH, Wang ST, et al.: Prognostic value of vascular endothelial growth factor expression in colorectal cancer patients. Eur J Cancer 36: 748-753, 2000

4. Pinedo HM, Slamon J: Translational Research: The role of VEGF in tumor angiogenesis. Oncologist 5: 1-2, 2000

5. Ferrara N: Role of Vascular Endothelial Growth Factor in physiologic and pathologic angiogenesis: therapeutic implications. Sem Oncol 29(6), (Suppl. 16): 10-14, 2002

6. Kabbinavar F, Hurwitz H, Fehrenbacher L, Meropol N, Novotny $\mathrm{W}$, Lieberman G, et al.: Phase II, randomized trial comparing bevacizumab plus fluorouracil (FU)(leucovorin (LV) with FU/LV alone in patients with metastatic colorectal cancer. J Clin Oncol 21(1): 60-65, 2003

7. Fong TA, Shawver LK, Sun L, et al.: SU5416 is a potent and selective inhibitor of the vascular endothelial growth factor receptor (Flk-1/KDR) that inhibits tyrosine kinase catalysis, tumor vascularization, and growth of multiple tumor types. Cancer Res 59: 99-106, 1999

8. Sukbuntherng J, Cropp G, Hannah A, Wagner G, Shawver L, Antonian L: Pharmacokinetics and Interspecies Scaling of a novel VEGF receptor inhibitor, SU5416. J Pharmacy Pharmacol 53: 1629-1636, 2001

9. Salzberg M, Pless M, Rochlitz C, Scigalla P, Herrmann R: A phase I and pharmacokinetic study of oral administration of SU5416 in patients with advanced solid tumors. Presentation at poster workshop session on Phase I/Clinical Pharmacology ECCO 2003, Copenhagen

10. Antonian L, Zhang H, Yang C, Wagner G, Shawver L, Shet M, et al.: Biotransformation of the Anti-Angiogenic compound SU5416. Drug Metab Disposit 28: 1505-1512, 2000

11. Ogilvie, Scheinkönig JA, Antonian L, Ye C, Tan W, Madan A, Parkinson A: SU5416 undergoes concerted (two-step) oxidation by human CYP1A2 to an alcohol and then the corresponding carboxylic. Drug Metab Rev 23(S1): 111, 2002

12. Internal Sugen Data onFile

13. Raeissi SD, Waltz K, Sukbuntherng J, Lipson K, Sweeney D, Kim TW, Antonian L: In vitro model to elucidate the potential of the receptor of the receptor tyrosine kinase (RTK) inhibitor SU5416 to induce CYP1A1. Proc Am Ass Cancer Res 42: 190, 2001

14. Solis WA, Wong SGW, Vela J, Antonian, Sweeney DJ: Investigation into the mechanism of CYP1A1 induction by indolinone receptor tyrosine kinase inhibitors. Presented in IUPHAR, San Francisco, CA, $7 / 2002$

15. Stoppeck A, Sheldon M, Vahedian M, Cropp G, Gosalia R, Hannah A: Results of a phase I dose-escalating study of the antiangiogenic agent, SU5416, in patients with advanced malignancies. Clin Cancer Res 8: 2798-2805, 2002

16. Mendel D, Schreck R, West DC, Li G, Strawn L, Tanciongco S, et al.: The angiogenesis inhibitor SU5416 has long lasting effects on VEGF receptor phosphorylation and function. Clin Cancer Res 6: 4848-4858, 2000

17. Laird A, Vajkoczy P, Shawver L, Thurnher A, Liang C, et al.: SU6668 is a potent anti-angiogenic and anti-tumor agent which induces regression of established tumors. Cancerv Res 60: 41524160,2000

Address for offprints: Marc Salzberg, Pharmacia Inc., Peapack, USA. 